\title{
Rectal Tuberculosis - A Complete Review
}

\author{
Nived Jayaraj $^{1}$, A. R. Shabaraya ${ }^{2}$ \\ ${ }^{1}$ Student, Department of Pharmacy Practice, Srinivas College of Pharmacy, Mangalore, Karnataka, \\ India - 574143 \\ ${ }^{2}$ Professor \& Head, Department of Pharmacy Practice, Srinivas College of Pharmacy, Mangalore, Karnataka, \\ India - 574143 \\ Corresponding Author: Nived Jayaraj
}

\begin{abstract}
Tuberculosis (TB) is a communicable disease caused mainly by Mycobacterium tuberculosis and its most frequent location is lung. Tuberculosis which affects the rectum is a rare extra-pulmonary sort of the disease that needs recognition because it requires specific treatment. Clinical and endoscopic presentations of intestinal TB are often almost like those of other diseases, like neoplasias and regional enteritis. Therefore, a high level of suspicion is required, as well as the performance of microbiologic studies, to confirm the diagnosis. Tuberculosis which affects the rectum is called rectal tuberculosis. Most common symptoms of rectal tuberculosis are abdominal pain, hematochezia, bowel habit change, episodes of rectal bleeding. The causative organisms of the disease include Mycobacterium tuberculosis and Bovis. Diagnosis of rectal tuberculosis is based on characteristic endoscopic appearance of lesions, histopathologic features of tuberculosis in biopsy/ resected material, and response to antitubercular therapy. It is still a great challenge even for experienced clinicians to diagnose the abdominal tuberculosis since it is an excellent mimicker that has unusual presentations. A high index of suspicion is important for reaching its diagnosis.
\end{abstract}

Keywords: Rectal tuberculosis,

Tuberculosis, Rectal TB

\section{INTRODUCTION}

Tuberculosis (TB) is a communicable disease caused by mycobacterium tuberculosis and its most frequent location is lung. Tuberculosis which affects the rectum is a rare extra-pulmonary sort of the disease that needs recognition because it requires specific treatment ${ }^{1}$. Extrapulmonary TB can affect the lymph nodes, the genitourinary tract, the osteoarticular system, the meninges, and also the digestive tract. Digestive involvement can present at any segment of the alimentary canal, and most often affects the ileocecal region.

The clinical and endoscopic presentations of intestinal TB are often almost like those of other diseases, like neoplasias and regional enteritis (CD). Therefore, a high level of suspicion is required, as well as the performance of microbiologic studies, to confirm the diagnosis.

\section{RECTAL TUBERCULOSIS}

Tuberculosis affecting the rectum is called rectal tuberculosis. Most common symptoms of rectal tuberculosis are abdominal pain, hematochezia (a condition in which blood is passed through anus, usually in or with stools), bowel habit change, episodes of rectal bleeding, as well as fatigue, anorexia, unquantified weight loss, and a sensation of fever ${ }^{2}$. In addition, intestinal obstruction, anorectal abscess and rectal fistula may also appear.

\section{CAUSES AND MODE OF SPREAD}

Main causes are Mycobacterium tuberculosis and Bovis. Various modes of spread include hematogenous spread from active pulmonary or miliary tuberculosis, swallowing of an infected sputum,ingestion 
of contaminated milk or food, through lymph channels from infected nodes and contiguous spread from adjacent organs.

\section{DIAGNOSIS}

Diagnosis of rectal tuberculosis is based on characteristic endoscopic appearance of lesions, histopathologic features of tuberculosis in biopsy/resected material, and response to antitubercular therapy $^{3}$. It is still a great challenge even for experienced clinicians to diagnose the abdominal tuberculosis. It is an excellent mimicker that has unusual presentations. A high index of suspicion is important for reaching its diagnosis ${ }^{4}$.

- Barium examination: A small bowel barium may show dilated loops, thickened folds or even stricture.

- Colonoscopy: May show ulcerative proliferative lesion involving part of rectal mucosa.

- CT abdomen: Computed tomography (CT) of the abdomen is a diagnostic imaging test. May reveal apparent wall thickening of the mild rectum.

- Rectal biopsy: A rectal biopsy is a procedure to remove a small piece of tissue from the rectum for examination it can detect the presence of Mycobacterium.

\section{TREATMENT}

WHO recommends 6-9 month course. Patient presents with complications and late presentations are also common in many cases. So, more often treatment for one year is required in these patients.

\begin{tabular}{|l|l|l|}
\hline DRUG & $\begin{array}{l}\text { DOSAGE } \\
(\mathbf{m g} / \mathbf{k g})\end{array}$ & ADVERSE EFFECTS \\
\hline Isoniazid & 5 & $\begin{array}{l}\text { PERIPHERAL NEURITIS, } \\
\text { hepatitis, fever, rashes }\end{array}$ \\
\hline Rifampicin & 10 & $\begin{array}{l}\text { HEPATITIS, orange urine, skin } \\
\text { rashes, flu syndrome, purpura }\end{array}$ \\
\hline Ethambutol & 15 & $\begin{array}{l}\text { OPTIC } \\
\text { NEURITIS,Hyperurecemia }\end{array}$ \\
\hline Pyrazinamide & 25 & $\begin{array}{l}\text { Hepatotoxicity, } \\
\text { hyperurecemia,arthralgia }\end{array}$ \\
\hline Streptomycin & 15 & $\begin{array}{l}\text { OTOTOXICITY, } \\
\text { NEPHROTOXICITY }\end{array}$ \\
\hline
\end{tabular}

\section{SECOND LINE DRUGS include}

- Amikacin

- Kanamycin

- Para aminosalicylic acid

- Ciprofloxacin

- Ofloxacin

- Levofloxacin

- Clarithromycin

- Azithromycin

- Rifabutin

In drug resistance cases secondline drugs are needed.

Directly observed therapy widely known as DOT is a technique used to ensure the person receiving and taking all medications as prescribed and to monitor response to treatment. It is widely used to manage tuberculosis disease. If facilities for directly observed therapy are available, initial treatment is the same as in short-term therapy ${ }^{5}$.

\section{CONCLUSION}

Rectal tuberculosis is probably the rarest site of gastrointestinal involvement. Gastrointestinal tuberculosis occurs mostly as a complication of pulmonary tuberculosis and rarely as a primary entity. Tuberculosis which affects the rectum is called rectal tuberculosis. Most common symptoms of rectal tuberculosis are abdominal pain, hematochezia, bowel habit change, episodes of rectal bleeding, as well as fatigue, anorexia, unquantified weight loss, and a sensation of fever. Rectal tuberculosis is common in developing countries; however, its diagnosis is difficult as it mimics Crohn's disease or malignancy. Mostly after clinical suspicion all patients are subjected to barium enema or CT scan which most of the times mimics malignancy on appearance.

Acknowledgement: None

Conflict of Interest: None

Source of Funding: None 


\section{REFERENCES}

1. Samarasekera D, Nanayakkara P. Rectal tuberculosis: A rare cause of recurrent rectal suppuration. Colorectal Disease [Internet]. 2008 [cited 9 June 2021];10(8):846-847. Available from: https://onlinelibrary.wiley.com/doi/abs/10.1 111/j.1463-1318.2008.01479.x

2. Gompertz M, Carreño L, Gil La Rotta L. Rectal tuberculosis: An uncommon clinical presentation and differential diagnosis with Crohn's disease. Revista de Gastroenterología de México (English Edition). 2019;84(4):524-526.

3. Puri A, Vij J, Chaudhary A, Kumar N, Sachdev A, Malhotra V et al. Diagnosis and outcome of isolated rectal tuberculosis.
Diseases of the Colon \& Rectum. 1996;39(10):1126-1129.

4. Abu-Zidan F, Sheek-Hussein M. Correction to: Diagnosis of abdominal tuberculosis: lessons learned over 30 years: pectoral assay. World Journal of Emergency Surgery. 2019;14(1).

5. Youtu.be. 2021. Before you continue to YouTube. [online] Available at: <https://youtu.be/1cURo8Pni_I> [Accessed 9 June 2021].

How to cite this article: Jayaraj N, Shabaraya AR. Rectal tuberculosis - a complete review. International Journal of Research and Review. 2021; 8(6): 260-262. DOI: https://doi.org/10. 52403/ijrr.20210631 